\title{
Design a CRLH Antenna for MIMO Applications with Single and Dual Band
}

\author{
Nada M. Khalil Al-Ani ${ }^{1 *}$, Oras A. Shareef Al-Ani', Mahmood F. Mosleh¹, Read A. Abd-Alhameed² \\ ${ }^{1}$ Department of Computer Engineering Techniques, Electrical and Electronic Technical Collage, Middle Techniqal University, \\ 10022 Al-Dora, Baghdad, Iraq \\ 2 School of Engineering and Informatics, University of Bradford, Central Admissions, BD7 1AZ Bradford, $51-53$ Hills Road, \\ United Kingdom \\ * Corresponding author, e-mail: eng.nada85@eetc.mtu.edu.iq
}

Received: 17 May 2020, Accepted: 07 January 2021, Published online: 05 July 2021

\begin{abstract}
A design of MIMO antenna with four elements each one consists of two polarized ports is proposed in this research. The design of each elements based on ZORA which is applied to get the advantage of band frequency flexibility. The proposed MIMO antenna is operated as a single and dual band by adjusting a ground structure. The resonance frequency is $3.9 \mathrm{GHz}$ for single band with bandwidth $1 \mathrm{GHz}$ (3.4-4.4) $\mathrm{GHz}$ at $-10 \mathrm{~dB}$ with $-27 \mathrm{~dB}$ isolation. The resonance frequencies for dual band are $4.95 \mathrm{and} 7 \mathrm{GHz}$ with bandwidth $1.23 \mathrm{GHz}$ (4.03-5.26) GHz and $410 \mathrm{MHz}$ (6.88-7.29) GHz at $-10 \mathrm{~dB}$ and less than $-12 \mathrm{~dB}$ isolation for both bands. The obtained size of FR-4 PCB for single band is $77 \times 150 \mathrm{~mm}^{2}$ while $82 \times 150 \mathrm{~mm}^{2}$ for dual band which are suitable for future smartphone.
\end{abstract}

\section{Keywords}

CRLH-TL, dual band, dual polarized antenna, tree structure, ZORA

\section{Introduction}

In 2002, the conception of composite right/left-handed (CRLH) transmission line (TL) metamaterials (MTMs) or planar negative-refractive index TL had been generalized to offer the implementations the practical applications for engineering in MTMs [1]. Such a paradigm has been exhibiting a rich potential possessing in electromagnetics engineering of the microwave devices with unusual properties [2]. The properties of CRLH are infinite-wavelength regime, leaky-wave radiation, bandpass behavior and nonlinear dispersion [2]. The last one makes CRLH exploiting the implementation of dual band antenna design [3]. The band allows the available space to be shared by a greater number of devices that back with benefit for wireless networks, at the same time increase the match of demand for high capacity in the cellular network [4]. With the future wireless communication, multiple input multiple output (MIMO) can achieve a high channel capacity [5]. However, the tradeoff between reducing the mutual coupling between antennas and low profile is the main challenge of MIMO antenna designing [6]. The polarization diversity considered as the best in antenna diversity as well as it can achieve a compact design [7]. In contrast, non-linear progressive phase, anti-parallel phase and group velocities which are the characteristics of lefthanded MTM-TL make it investigate the compact antenna design [8-10]. Novel zeroth-order resonator antenna (ZORA) is an example of CRLH -TL since its electrical length is zero, so antenna size can be more reduced than conventional antennas [11]. However, enormous research has been studied to reduce the mutual coupling between antenna elements of MIMO systems with reducing space between them such as metamaterial polarization-rotator (MPR) wall [12] and partial ground [13]. On the other hand, the researcher of [14, 15] are used and discussed the defected ground structure (DGS) and tree structure respectively. In this paper, a compact of dual polarized ports each with only one cell of left-handed transmission line with a tree structure for decoupling. The decoupling structure innovation proposed in this research is not only to improve the good isolation but also can generalize a dual band's frequency which will improve the usage of space in mobile devices.

\section{Microstrip ZORA theory}

Due to the dual ideal model of LH/RH TLs, the equivalent circuit of a one-unit cell of CRLH TL which shown in 
Fig. 1 (a) and (b). It consists of series resonators $\hat{L}_{R}$ and $\hat{C}_{L}$, shunt resonators $\hat{C}_{R}$ and $\hat{L}_{C}$ right and left-handed, respectively. It is worth to mention that the operating wavelength should be greater than the dimension of which unit cell $\hat{P}$. The compilation of such a circuit is LH-TL domination with low frequency while RH-TL is dominated at high frequency [16-19].

As shown in Fig. 1(a), the gab is zero between LH and $\mathrm{RH}$ regions, so that, it would satisfy the condition of balance which means $\hat{W}_{L}$ and $\hat{W}_{R}$ are the resonance frequencies of LH and RH, respectively will be equal, which can be given by [16]:

$\hat{W}_{L}=\frac{1}{\sqrt{\hat{L}_{L} \hat{C}_{L}}}$ and $\hat{W}_{R}=\frac{1}{\sqrt{\hat{L}_{R} \hat{C}_{R}}}$.

Besides, LH and RH resonance frequency will be equal to the resonance frequency of the TL where the CRLH-TL frequency can be written as [16]:

$\hat{W}_{0}^{2}=\hat{W}_{C L} \hat{W}_{C R}=\hat{W}_{L} \hat{W}_{R}=\frac{1}{\sqrt{\hat{L}_{L} \hat{L}_{R} \hat{C}_{L} \hat{C}_{R}}}$,

while the $\hat{W}_{C L}$ and $\hat{W}_{C R}$ represent the CRLH cut off a frequency that can be shown as [17]:

$$
\left.\begin{array}{l}
\hat{W}_{C L}=\hat{W}_{R}\left(\sqrt{1+\frac{\hat{W}_{L}}{\hat{W}_{R}}}-1\right) \\
\hat{W}_{C R}=\hat{W}_{R}\left(\sqrt{1+\frac{\hat{W}_{L}}{\hat{W}_{R}}}+1\right)
\end{array}\right\} .
$$

From another view, at the resonance frequency, the progressive phase of the electrical length which is the summation of RH and LH sections will be zero which is the result of usage microstrip ZORA. As a lot, the impedance of the CRLH-TL $\hat{Z}_{E}$ will be equal to both $\hat{Z}_{L}$ and $\hat{Z}_{R}$ which are the impedance of the left and right-handed respectively. $\hat{Z}_{L}$ and $\hat{Z}_{R}$ can be given by [16]:

$\hat{Z}_{L}=\sqrt{\frac{\hat{L}_{L}}{\hat{C}_{L}}}$ and $Z_{R}=\sqrt{\frac{\hat{L}_{R}}{\hat{C}_{R}}}$.

Accordingly, when the balance condition investigates the $\hat{W}_{C L}, \hat{W}_{C R}$, and $\hat{Z}_{E}$ will be fixed, thus making the unique configuration of the CRLH is illustrated as follow [17]:

$$
\left.\begin{array}{c}
\hat{W}_{0} \hat{C}_{L}=\frac{1}{\hat{W}_{0} \hat{C}_{L}}=\frac{\hat{W}_{0}}{\hat{W}_{C R}-\hat{W}_{C L}} 2 \hat{Z}_{E} \\
\hat{W}_{0} \hat{C}_{R}=\frac{1}{\hat{W}_{0} \hat{L}_{L}}=\frac{\hat{W}_{0}}{\hat{W}_{C R}-\hat{W}_{C L}} \frac{2}{\hat{Z}_{E}}
\end{array}\right\} .
$$

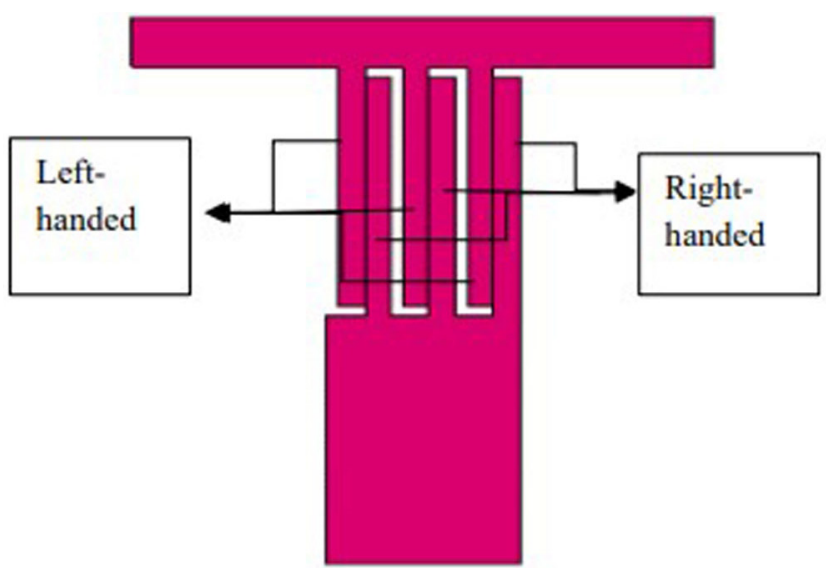

(a)

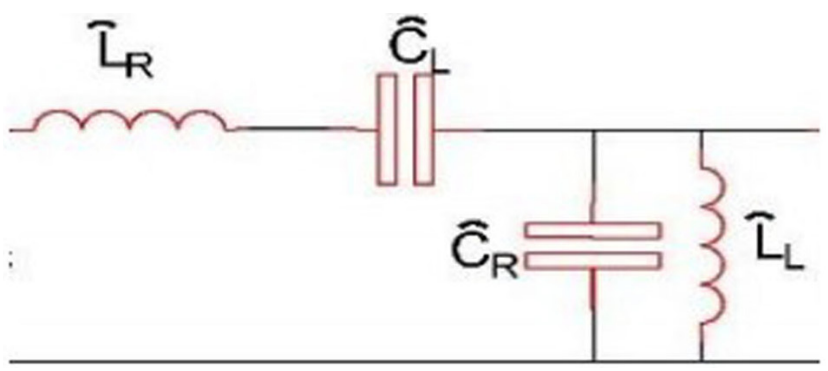

(b)

Fig. 1 (a) 2D layout of CRLH-TL; (b) Equivalent circuit model of CRLH-TL.

Also, because of the non - linearity of the LH which represents the negative part and the linearity of RH section, the propagation will be constant which can be determined respectively for both sections as follow [19]:

$$
\hat{K}_{L}=-\frac{1}{\left(\hat{W}_{0} \sqrt{\hat{L}_{L} \hat{C}_{L}}\right)} \text { and } \hat{K}_{R}=\left(\hat{W}_{0} \sqrt{\hat{L}_{L} \hat{C}_{L}}\right) \text {. }
$$

\section{Single antenna design and results}

In this research, the first step of the design procedure is the single antenna element simulation and the results obtained are an evaluation before going to design MIMO prototype. By considering the aim of this research, it must keep in mind the frequency band of the single element which must much the corresponding band of MIMO system. Fig. 2 shows the proposed paradigm which consists of three layers. The patch layer consists of a dual polarized ZORA feeding with $50 \Omega$ connected by SMA. Such a patch is printed on a dielectric substrate layer with thickness $1.6 \mathrm{~mm}$ made of FR-4 with relative permittivity 4.4 and loss tangent 0.025 . 


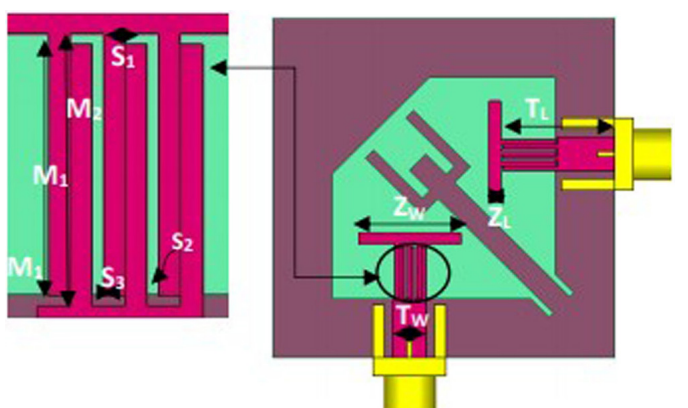

(a)

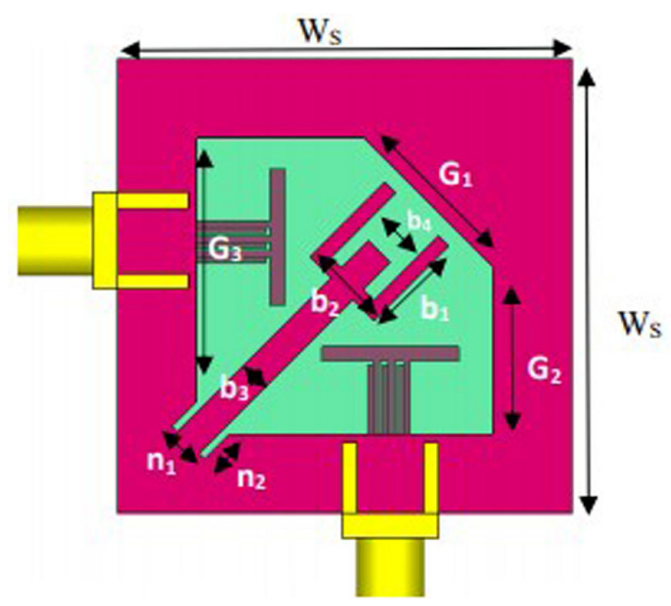

(b)

Fig. 2 (a) Single antenna element front view; (b) Single antenna element back view.

\subsection{Single band}

Fig. 2 shows the geometrical layout of the single antenna element proposal and all the dimensions detailed in Table 1.

The result of S-parameter for the above model is illustrated in Fig. 3(a) which having a reflection coefficient about $-24 \mathrm{~dB}$ at $3.9 \mathrm{GHz}$ with coverage bandwidth about $1 \mathrm{GHz}$ (3.4-4.4) $\mathrm{GHz}$ at $-10 \mathrm{~dB}$ and a good mutual coupling approach to $-27 \mathrm{~dB}$. On the other hand, VSWR which determines the antenna performance if have a value $<2$ as mentioned in [20] shown in Fig. 3(b) for both ports 1 and 2.

\begin{tabular}{lccc}
\multicolumn{4}{c}{ Table 1 Parameter values of the single element dimension. } \\
\hline Parameter & Value $(\mathrm{mm})$ & Parameter & Value $(\mathrm{mm})$ \\
\hline$Z_{W}$ & 9 & $G_{1}$ & 12 \\
$Z_{L}$ & 1 & $G_{2}$ & 11.01 \\
$T_{W}$ & 3 & $G_{3}$ & 17.38 \\
$T_{L}$ & 10 & $b_{1}$ & 7 \\
$M_{1}$ & 4.80 & $b_{2}$ & 6 \\
$M_{2}$ & 5 & $b_{3}$ & 2 \\
$S_{1}$ & 0.6 & $b_{4}$ & 4 \\
$S_{2}$ & 0.2 & $n_{1}$ & 2.30 \\
$S_{3}$ & 0.4 & $n_{2}$ & 3 \\
$W_{S}$ & 30 & & \\
\hline
\end{tabular}

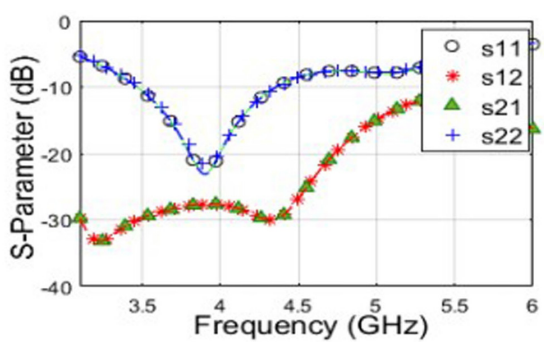

(a)

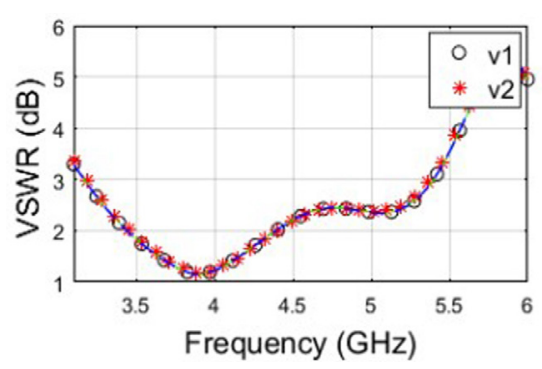

(b)

Fig. 3 (a) S-parameter of single band model; (b) VSWR of single band model.

Also, 2-D polar plot for E-field and H-field which is mentioned as radiation pattern shown in Fig. 4(a) and (b), respectively, which determines that such a single element model has a bidirectional radiation pattern with angular lobe $96.3 \mathrm{deg}$. Fig. 4(c) shows the 3D view which exhibits the radiation pattern has been covering the top and bottom of the single element prototype. It is worth to mention that the aim of the proposal of $[21,22]$ are to employ spatial diversity. In contrast, the current model has applied the polarization diversity to match the MIMO system for the smartphone application.

\subsection{Dual band}

To obtain a dual band frequency. Some modification has been applied on the single antenna element which proposed in Subsection 3.1. Such modification included some variation in the dimensions of the ground layer which converts the etching of the tree shape to stub shape which has clear out in Fig. 5. As a note, all the proposed dimensions in $\mathrm{mm}$.

As a result, a dual band frequencies are obtained, which are 4.7 and $7.08 \mathrm{GHz}$ with return losses of $-23 \mathrm{db}$ and $-21 \mathrm{~dB}$ respectively. Furthermore, the bandwidths for both points are $1.23 \mathrm{GHz}(4.03-5.26) \mathrm{GHz}$ and $410 \mathrm{MHz}(6.88-7.29)$ $\mathrm{GHz}$ at $-10 \mathrm{~dB}$. The mutual coupling for the proposed paradigm is less than $-12 \mathrm{~dB}$. From another view, the VSWR not approach 2 with both resonance frequency points, all results are shown in Fig. 6. Also, because of the $4.7 \mathrm{GHz}$ which is the first point has a larger bandwidth than the second point 
Farfield E-Pattern Abs (Phi=0)

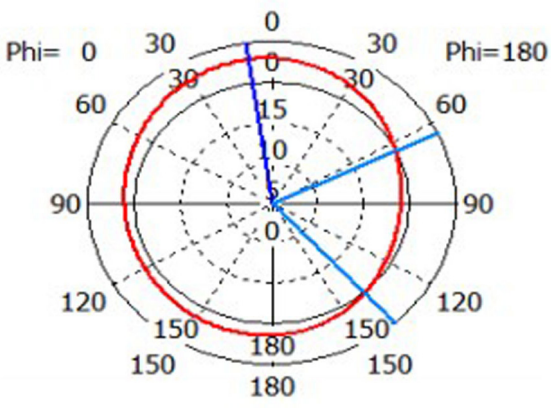

Theta / Degree vs. dBV

(a)

Farfield E-Pattern Abs (Phi=90)

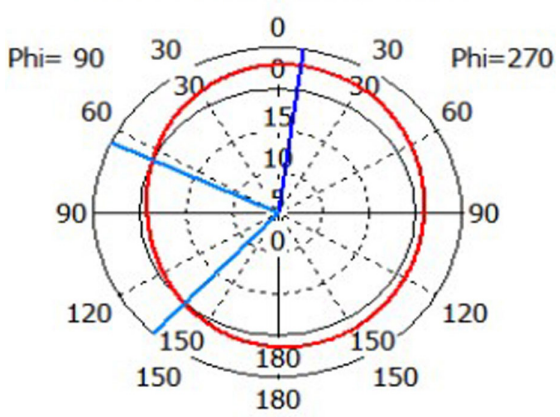

Theta / Degree vs. dBV

(b)

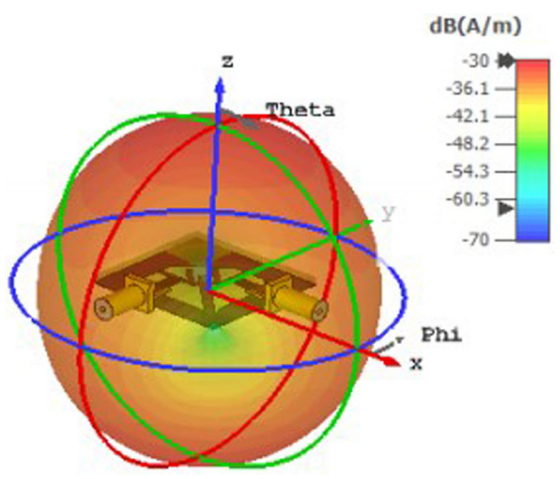

(c)

Fig. 4 (a) Radiation pattern of single band model E-field; (b) Radiation pattern of single band model H-field; (c) 3D-view.

which has $7.08 \mathrm{GHz}$ frequency. As a result, the 2-D polar pattern of the first one has more size of radiation pattern as shown in Fig. 7(a) and (b) with red color line, however, both points have bidirectional patterns. Also, the 3D view in Fig. 7(b) and (c) is shown the radiation pattern is covered allover directions of the single element.

\section{MIMO array and results}

To obtain a MIMO prototype, four of the proposed single antenna elements are installed on the corners of printed

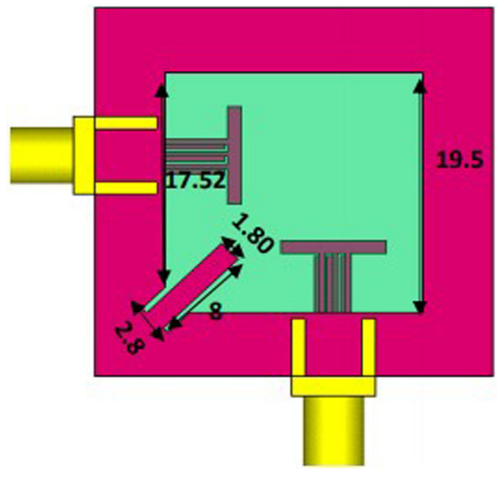

Fig. 5 Another ground geometry of the single element antenna.

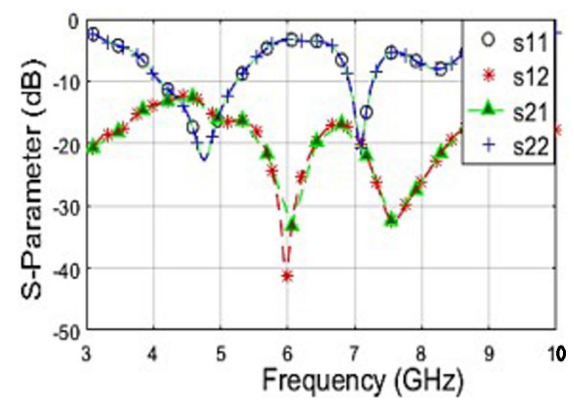

(a)

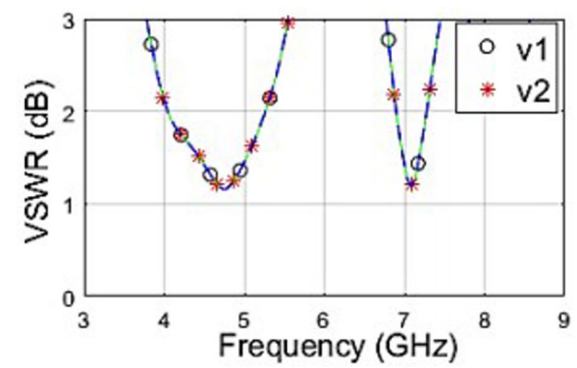

(b)

Fig. 6 (a) S-Parameter results of the dual band of the single element; (b) VSWR results of the dual band ofthe single element.

circuit board (PCB). Such PCB is fabricated from FR-4 with a characteristic mentioned in Section 3 which has a dimension (length and width) that has been obtained with many trying methods.

\subsection{Single band}

The proposed single element model in Section 3 has installed with $77 \times 150 \mathrm{~mm}^{2}$ of PCB as shown in Fig. 8 .

The results of which MIMO system proposed is shown in Fig. 9, which determines that all ports can operate at the same resonance frequency which matches the bandwidth of a single antenna element. However, such MIMO model has return losses $S_{n m}-50 \mathrm{~dB}$ of some group of ports while others 


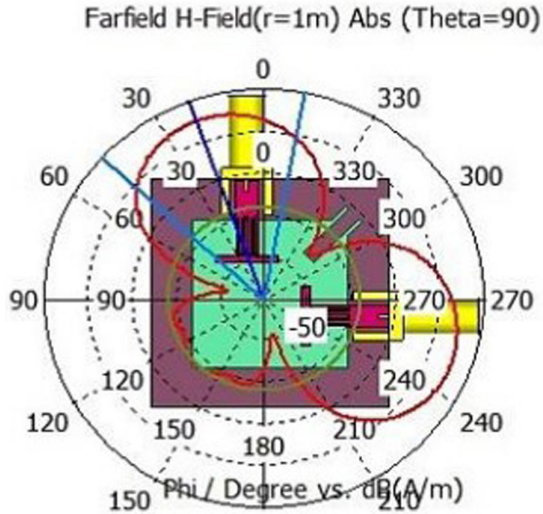

(a)

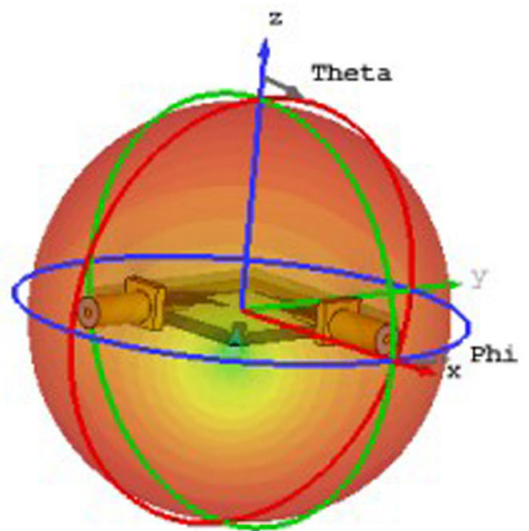

(c)
Farfield $\mathrm{H}$-Field $(\mathrm{r}=1 \mathrm{~m})$ Abs (Theta $=90)$

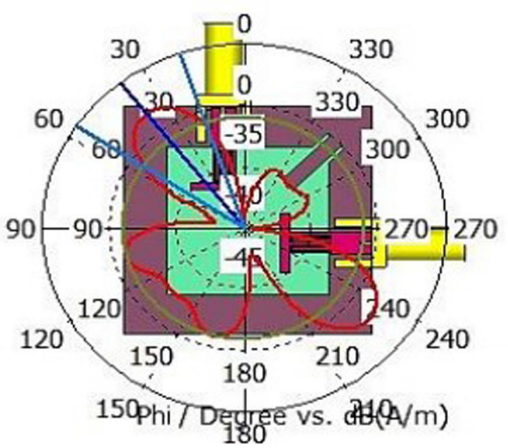

(b)

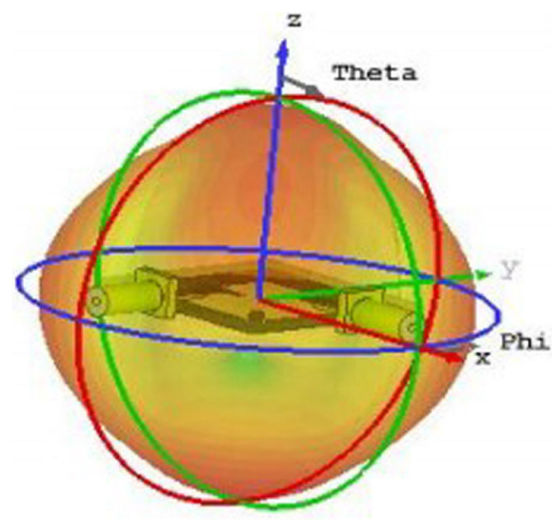

(d)

Fig. 7 (a) 2D polar radiation pattern of the single element at $4.55 \mathrm{GHz}$; (b) 2D polar radiation pattern of the single element at $7 \mathrm{GHz}$; (c) 3D view radiation pattern of the single element at $4.55 \mathrm{GHz}$; (d)

$3 \mathrm{D}$ view radiation pattern of the single element at $7 \mathrm{GHz}$.

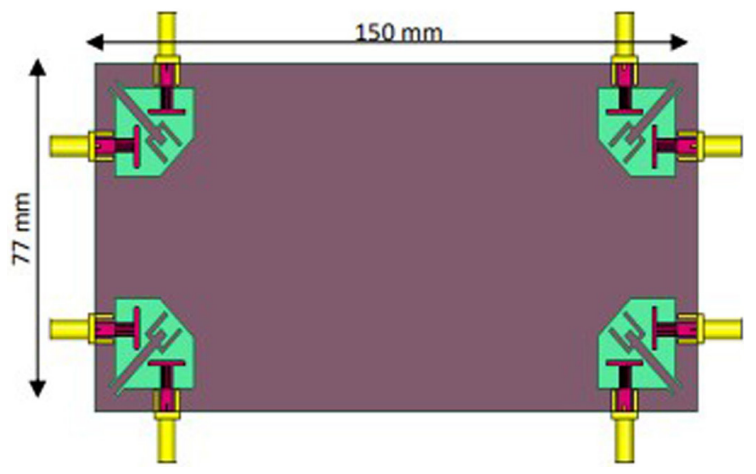

(a)

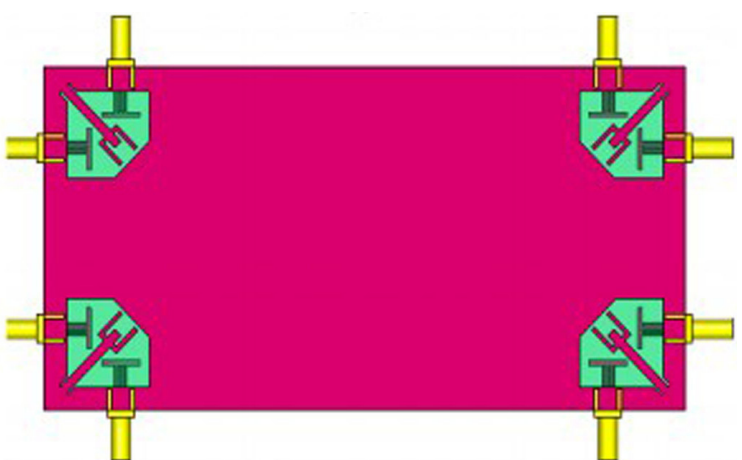

(b)

Fig. 8 (a) Front view of MIMO antenna system; (b) Back view of MIMO antenna system.

have about $-40 \mathrm{~dB}$. As well as, the mutual coupling $S_{n f}$ is less than $-20 \mathrm{~dB}$ for all ports over the operating frequency bandwidth and the VSWR less than 2 for all ports. Also, Fig. 10 shows the radiation pattern of MIMO system which obviously in 2D polar the radiation can cover all directions of the PCB with angular lobe of $32 \mathrm{deg}$. In addition, the $3 \mathrm{D}$ view exhibits the radiation cover the top and bottom of the device body with H-max. $-22.2 \mathrm{~dB}$ and gain $5.5 \mathrm{dBi}$.

\subsection{Dual band}

In contrast, to obtain a dual band of MIMO array, the proposed single element antenna detailed in Subsection 3.2 is arranged with $82 \times 150 \mathrm{~mm}^{2}$ of PCB as shown in Fig. 11 .

The simulation results of the proposed MIMO system shown in Fig. 12 which confirms that the MIMO ports can be operated with two band frequencies of 4.95 and $7 \mathrm{GHz}$ with $S_{n m}-16$ and $-26.5 \mathrm{~dB}$ respectively. The coverage 


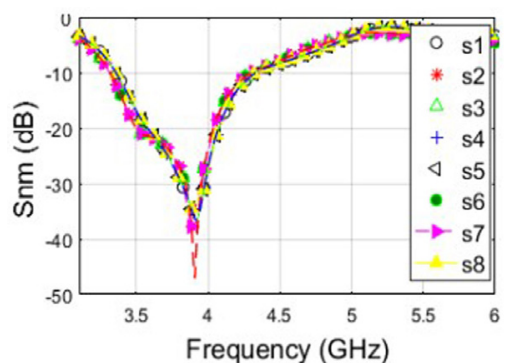

(a)

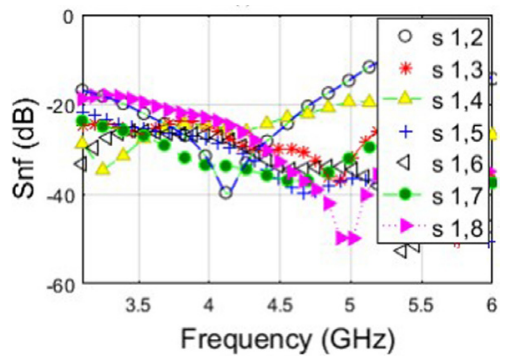

(b)

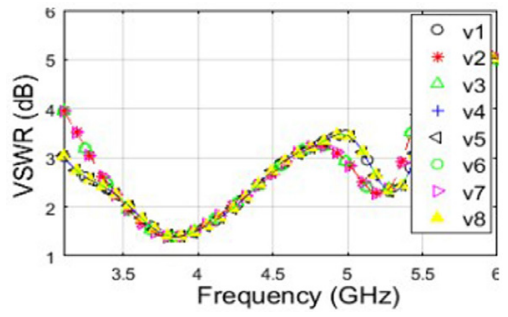

(c)

Fig. 9 (a) Snm results of MIMO array;

(b) $S_{n f}$ results of MIMO array; (c) VSWR results of MIMO array.

bandwidths have been obtained matching the same as the frequency bands in Subsection 3.2 with shifted resonance frequency mentioned above. Sab and VSWR have been obtained less than -12 and $2 \mathrm{~dB}$ respectively for both operating frequency bandwidths.

In the term of the radiation pattern, Fig. 13 shows the $2 \mathrm{D}$ patterns can be seen in omni-direction view for both resonance frequency points. Due to large operating frequency bandwidth of the first frequency point it seems to have more radiation coverage area. As a lot, both radiations having 15.8 and $9.4 \mathrm{deg}$. The angular width of the lobe.

Another viewing of the radiation pattern can be exhibit as a 3D which illustrated in Fig. 14 for two resonance frequency operating points. It clears out as a bubble covering the top and bottom and all directions of the mobile device with H-max $-22.6,-20.6 \mathrm{~dB}$ and 5.62, $7.12 \mathrm{dBi}$ of gain, respectively.

To evaluate this model, Table 2 depicts the difference between current model with other references [21-25].

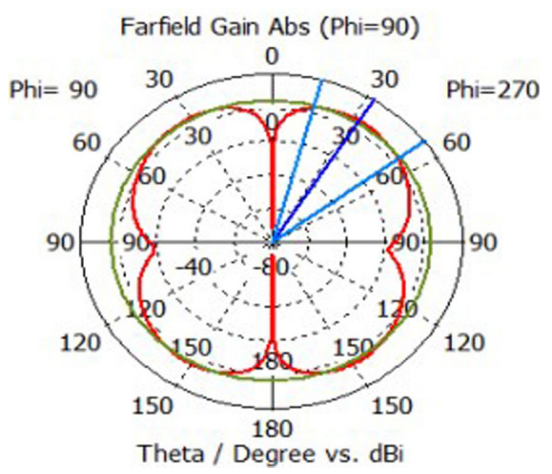

(a)

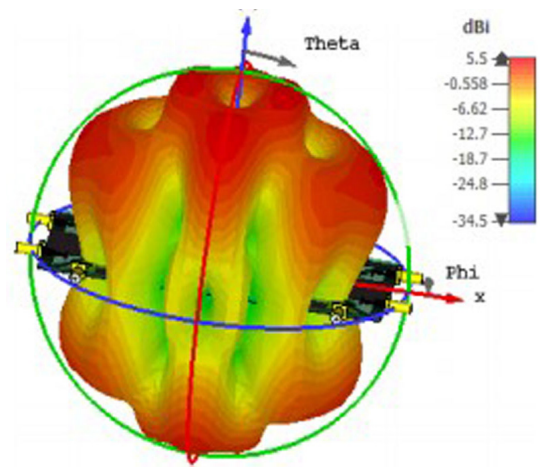

(b)

Fig. 10 (a) 2D radiation pattern of MIMO with single band; (b) 3D radiation pattern of MIMO with single band.

It seems that this model has the desired characteristics with a good size of PCB which represents the mother smartphone. The higher number of ports means higher data rate which is desired for future wireless communication.

\section{Conclusion}

In this paper, a design of MIMO antenna based on ZORA model. The MIMO prototype consists of four elements installed on the corner of the rectangular FR-4 PCB. The proposed model operated as a single band of $3.9 \mathrm{GHz}$ when the ground based on a tree structure. Also, the proposed model operated as a dual band with operating frequency 4.95 and $7 \mathrm{GHz}$ when the ground geometry modified into stub structure. The bandwidth of the single band model is $1 \mathrm{GHz}(3.4-4.4) \mathrm{GHz}$ at $-10 \mathrm{~dB}$ with $-27 \mathrm{~dB}$ of mutual coupling. While the bandwidths of dual band model are $1.23 \mathrm{GHz}(4.03-5.26) \mathrm{GHz}$ and $410 \mathrm{MHz}(6.88-7.29)$ $\mathrm{GHz}$ at $-10 \mathrm{~dB}$ with a mutual coupling less than $-12 \mathrm{~dB}$ for both bands. The proposed single band MIMO antenna has the advantage of a suitable size $(77 \times 150) \mathrm{mm}^{2}$ while the dual band with a size of $(82 \times 150) \mathrm{mm}^{2}$ which matches the size of smart phone operated for future wireless communication. 


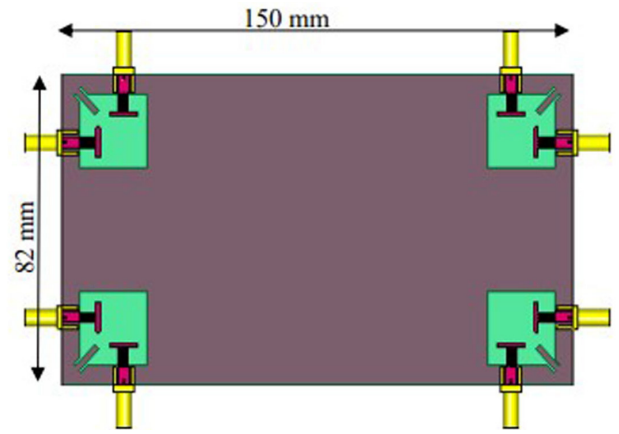

(a)

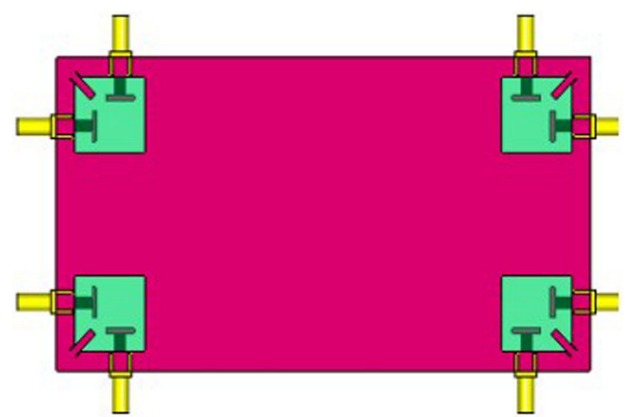

(b)

Fig. 11 (a) Front view of MIMO antenna system; (b) Back view of MIMO antenna system.

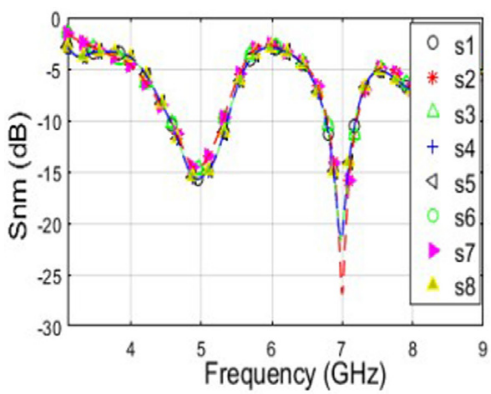

(a)

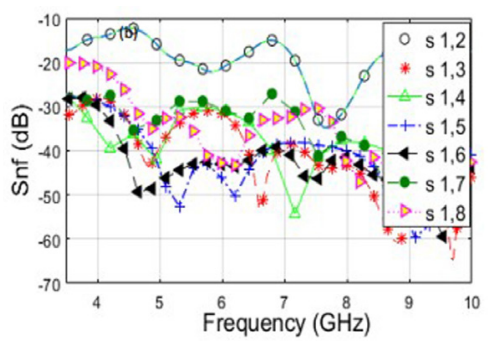

(b)

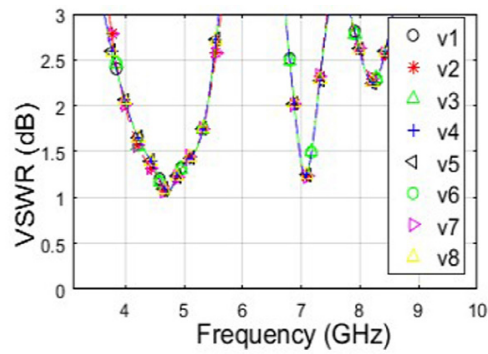

(c)

Fig. 12 (a) $S_{n m}$ MIMO antenna system results; (b) $S_{a b}$ MIMO antenna system results; (c)VSWR MIMO antenna system results. 
Farfield Gain Abs (Phi $=90)$

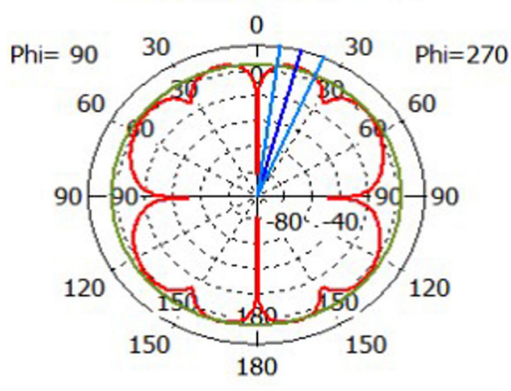

Theta / Degree vs. dBi

(a)

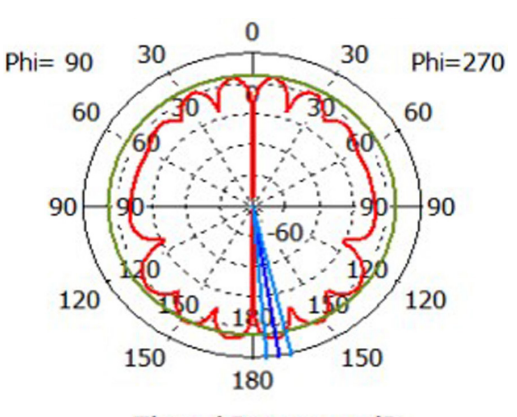

Theta / Degree vs. dBi

(b)

Fig. 13 (a) The 2D polar radiation pattern at $4.55 \mathrm{GHz}$; (b) The 2D polar radiation pattern at $7 \mathrm{GHz}$.

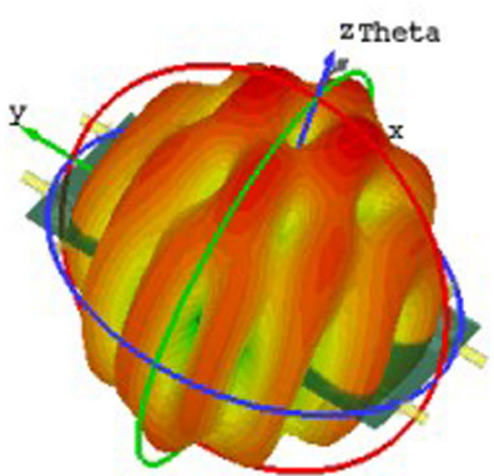

dBi

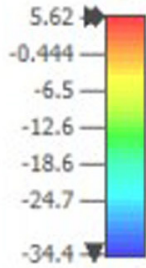

(a)

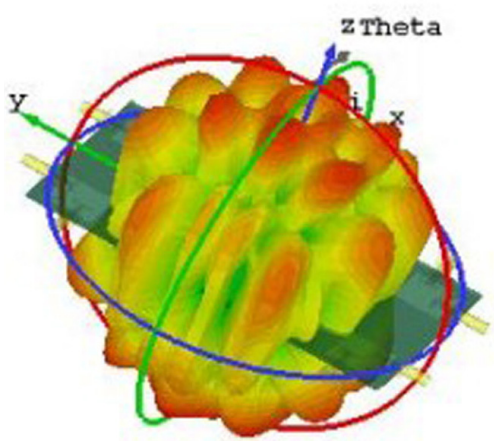

$\mathrm{dBi}$

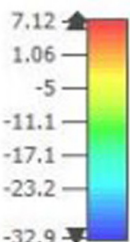

(b)

Fig. 14 (a) 3D view of of MIMO antenna system radiation pattern at $4.55 \mathrm{GHz}$; (b) 3D view of of MIMO antenna system radiation pattern at $7 \mathrm{GHz}$.

Table 2 Difference between currently model and previous references.

\begin{tabular}{lcccccc}
\hline Ref. & $\mathrm{D}\left(\mathrm{mm}^{3}\right)$ & $S_{n f}(\mathrm{~dB})$ & $\mathrm{BW}(\mathrm{GHz})$ & $\begin{array}{c}\text { Applied } \\
\text { Technique }\end{array}$ & $\begin{array}{c}\text { Type of } \\
\text { Diversity }\end{array}$ & NO. ports \\
\hline$[21]$ & $65 \times 22.5 \times 5$ & -40 & $9.7-12.3$ & MTM-EBG & Spatial & 3 \\
{$[22]$} & $40 \times 20 \times 5$ & -13.5 & $1.7-3.66$ & Meta-surface & Spatial & 2 \\
{$[23]$} & $23 \times 23 \times 1.6$ & -37 & 5 & Fractal & Spatial & 2 \\
{$[24]$} & $14.5 \times 9 \times 1.6$ & -13.5 & $139-141$ & MSWI & Spatial & 2 \\
{$[25]$} & $120 \times 50 \times 1.6$ & $<-20$ & $9.55-10.81$ & Slots & Spatial & 2 \\
This work & $150 \times 77 \times 1.6$ & $<-20$ & $(3.4-4.4)$ & MTM & Polarization & 8 \\
$(1)$ & & & $(4.03-5.26)$ & & & \\
This work & $180 \times 82 \times 1.6$ & $<-20$ & $(6.88-7.29)$ & MTM & Polarization & 8 \\
$(2)$ & & & &
\end{tabular}

\section{References}

[1] Aznar, F., Gil, M., Bonache, J., Martín, F. "Modelling metamaterial transmission lines: a review and recent developments", OptoElectronics Review, 16(3), pp. 226-236, 2008. https://doi.org/10.2478/s11772-008-0028-x

[2] Caloz, C., Itoh, T. "Electromagnetic Metamaterials: Transmission Line Theory, and Microwave Applications", John Wiley \& Sons, Hoboken, NJ, USA, 2005.
[3] Otto, S., Rennings, A., Caloz, C., Waldow, P., Itoh, T. "Composite right/left-handed /spl lambda/-resonator ring antenna for dual-frequency operation", In: 2005 IEEE Antennas and Propagation Society International Symposium, Washington, DC, USA, 2005, pp. 684-687. https://doi.org/10.1109/APS.2005.1551413

[4] Yeom, I., Bae Jung, Y., Won Jung, C. "Wide and Dual-Banded MIMO Antenna with Omnidirectional and Directional Radiation Patterns for Indoor Access Points", Journal of Electromagnetic Engineering and Science, 19(1), pp. 20-30, 2019.

https://doi.org/10.26866/jees.2019.19.1.20 
[5] Halperin, D., Hu, W., Sheth, A., Wetherall, D. "802.11 with multiple antennas for dummies", ACM SIGCOMM Computer Communication Review, 40(1), pp. 19-25, 2010. https://doi.org/10.1145/1672308.1672313

[6] Molisch, A. F., Win, M. Z. "MIMO systems with antenna selection", IEEE Microwave Magazine, 5(1), pp. 46-56, 2004.

https://doi.org/10.1109/MMW.2004.1284943

[7] Antenova, Queen of Mary, University of London "Antenna Designs for MIMO Systems", Her Majesty's Stationery Office (HMSO), London, UK, 2004.

[8] Al-Ani, N. M. K., Al-Ani, O. A. S., Mosleh, M. F., Abd-Alhameed, R. A. "A Design of MIMO Prototype in C-Band Frequency for Future Wireless Communications", Advanced Electromagnetics, 9(1), pp. 78-84, 2020.

https://doi.org/10.7716/aem.v9i1.1333

[9] Eleftheriades, G. V., Balmain, K. G. "Negative-Refraction Metamaterials: Fundamental Principles and Applications", John Wiley \& Sons, Inc., Hoboken, NJ, USA, 2005.

[10] Abdalla, M., Abdelnaby, U., Mitkees, A. A. "Compact and triple band meta-material antenna for all WiMAX applications", In: 2012 International Symposium on Antennas and Propagation (ISAP), Nagoya, Japan, 2012, pp. 1176-1179.

[11] Leong, K. M. K. H., Lee, C. J., Itoh, T. "Compact Metamaterial Based Antennas for MIMO Applications", In: 2007 International workshop on Antenna Technology: Small and Smart Antennas Metamaterials and Applications, Cambridge, UK, 2007, pp. 87-90. https://doi.org/10.1109/IWAT.2007.370086

[12] Farahani, M., Pourahmadazar, J., Akbari, M., Nedil, M., Sebak, A. R., Denidni, T. A. "Mutual Coupling Reduction in Millimeter-Wave MIMO Antenna Array Using a Metamaterial Polarization-Rotator Wall", IEEE Antennas and Wireless Propagation Letters, 16, pp. 2324-2327, 2017. https://doi.org/10.1109/LAWP.2017.2717404

[13] Shandal, S. A., Mezaal, Y. S., Mosleh, M. F., Kadim, M. A. "Miniaturized Wideband Microstrip Antenna for Recent Wireless Applications", Advanced Electromagnetics, 7(5), pp. 7-13, 2018. https://doi.org/10.7716/aem.v7i5.806

[14] Abdalla, M. A., Ibrahim, A. A. "Compact and Closely Spaced Metamaterial MIMO Antenna With High Isolation for Wireless Applications", IEEE Antennas and Wireless Propagation Letters, 12, pp. 1452-1455, 2013. https://doi.org/10.1109/LAWP.2013.2288338

[15] Zhang, S., Ying, Z., Xiong, J., He, S. "Ultrawideband MIMO/ Diversity Antennas With a Tree-Like Structure to Enhance Wideband Isolation", IEEE Antennas and Wireless Propagation Letters, 8, pp. 1279-1282, 2009. https://doi.org/10.1109/LAWP.2009.2037027

[16] Yoo, S. "Advanced Metamaterial Circuits for Microwave and Millimeter Wave Applications", PhD dissertation, Florida University, 2018.
[17] Alibakhshikenari, M., Virdee, B. S., See, C. H., Abd-Alhameed, R., Falcone, F., Limiti, E. "Mutual-Coupling Reduction in Metamaterial Substrate Integrated Waveguide Slotted Antenna Arrays Using Metal Fence Isolators for SAR and MIMO Applications", In: 2018 12th International Congress on Artificial Materials for Novel Wave Phenomena (Metamaterials), Espoo, Finland, 2018, pp. 013-015. https://doi.org/10.1109/MetaMaterials.2018.8534166

[18] Liu, C., Huang, K. "Metamaterial Transmission Line and its Applications", In: Mukherjee, M. (ed.) Advanced Microwave and Millimeter Wave Technologies: Semiconductor Devices Circuits and Systems, InTech, Vukovar, Croatia, 2010, pp. 249-274. https://doi.org/10.5772/8770

[19] Alibakhshikenari , M., Virdee, B. S., Ali, A., Limiti, E. "Miniaturised planar-patch antenna based on metamaterial L-shaped unit-cells for broadband portable microwave devices and multiband wireless communication systems", IET Microwaves, Antennas \& Propagation, 12(7), pp. 1080-1086, 2018. https://doi.org/10.1049/iet-map.2016.1141

[20] Shandal, S., Mezaal, Y. S., Kadim, M., Mosleh, M. "New Compact Wideband Microstrip Antenna for Wireless Applications", Advanced Electromagnetic, 7(4), pp. 85-92, 2018. https://doi.org/10.7716/aem.v7i4.860

[21] Alibakhshikenari, M., Vittori, M., Colangeli, S., Virdee, B. S., Andújar, A., Anguera, J., Limiti, E. "EM isolation enhancement based on metamaterial concept in antenna array system to support full-duplex application", In: 2017 IEEE Asia Pacific Microwave Conference (APMC), Kuala Lumpur, Malaysia, 2017, pp. 740-742. https://doi.org/10.1109/APMC.2017.8251553

[22] Alibakhshikenari, M., Virdee, B. S., Khalily, M., See, C. H., AbdAlharueed, R., Falcone, F., Limiti, E. "A New Study to Suppress Mutual-Coupling Between Waveguide Slot Array Antennas Based on Metasurface Bulkhead for MIMO Systems", In: 2018 AsiaPacific Microwave Conference (APMC), Kyoto, Japan, 2018, pp. 500-502. https://doi.org/10.23919/APMC.2018.8617287

[23] Alibakhshikenari, M., Khalily, M., Virdee, B. S., See, C. H., Abd-Alhameed, R. A., Limiti, E. "Mutual Coupling Suppression Between Two Closely Placed Microstrip Patches Using EM-Bandgap Metamaterial Fractal Loading", IEEE Access, 7, pp. 23606-23614, 2019 . https://doi.org/10.1109/ACCESS.2019.2899326

[24] Alibakhshikhenari, M., Virdee, B. S., Shukla, P., See, C. H., Abd-Alhameed, R., Falcone, F., Limiti, E. "Meta-Surface Wall Suppression of Mutual Coupling between Microstrip Patch Antenna Arrays for THz-Band Applications", Progress In Electromagnetics Research Letters, 75, pp. 105-111, 2018.

[25] Alibakhshikenari, M., Salvucci, A., Polli, G., Virdee, B. S., See, C. H., Abd-Alhameed, R., Falcone, F., Andújar, A., Anguera, J., Limiti, E. "Mutual coupling reduction using metamaterial supersubstrate for high performance \& densely packed planar phased arrays", In: 2018 22nd International Microwave and Radar Conference (MIKON), Poznan, Poland, 2018, pp. 675-678. https://doi.org/10.23919/MIKON.2018.8405323 